\title{
Erratum to: Modified capillary electrophoresis based measurement of the binding between DNA aptamers and an unknown concentration target
}

Yue-Wei Zhang • Hai-Yu Yan • Ping Fu • Fei Jiang •

Yao Zhang • Wen-Xue Wu • Jin-Xiang Li

Received: 28 January 2014 / Accepted: 28 January 2014 / Published online: 12 February 2014

(C) Springer-Verlag Berlin Heidelberg 2014

Erratum to: Anal Bioanal Chem

DOI 10.1007/s00216-013-6968-0

The authors would like to call the reader's attention to the following:

Unfortunately Reference 17 in the article was cited unnecessarily.

Equation 2 should read:

$\frac{K_{d}\left(1+R_{1}\right)+[\mathrm{Apt}]_{1}}{K_{d}\left(1+R_{2}\right)+[\mathrm{Apt}]_{2}}=\frac{1+1 / R_{1}}{1+1 / R_{2}}$

The online version of the original article can be found at http://dx.doi. org/10.1007/s00216-013-6968-0.

Y.-W. Zhang $\cdot$ H.-Y. Yan $\cdot$ P. Fu $\cdot$ F. Jiang $\cdot$ Y. Zhang $\cdot$

W.-X. Wu $(\bowtie)$

Key Laboratory of Animal Epidemiology and Zoonosis, Ministry of

Agriculture, College of Veterinary Medicine, China Agricultural

University, Beijing, China

e-mail:wuwenxue@cau.edu.cn

Y. Zhang

e-mail: yao880413@163.com

J.-X. Li $(\bowtie)$

Chinese Academy of Agricultural Sciences, Beijing, China

e-mail: labboard@126.com 